\title{
The purpose and semantics of "combat" drilled stone axes-hammers
}

\begin{abstract}
The article attempts to study the semantics of the so-called "combat" drilled stone hammer axes (CDSHA) of the early and middle Bronze Age. Analysis of the morphology, materials, and appearance (decor) shows that the CDSHA mainly performed ritual functions. The decor on status items of this type (for example, axes-hammers from the Trojan collection L, etc.) has a pronounced calendar character.
\end{abstract}

Keywords: combat drilled stone axe-hammer, troy, collection L
Volume 5 Issue 2 - 2020

\author{
GSVrtanesyan \\ Center for the study of religions, Russian state University for \\ the Humanities, Russia

\begin{abstract}
Correspondence: GSVrtanesyan, Center for the study of religions, Russian state University for the Humanities, Russia, Email veges20II@yandex.ru
\end{abstract}

Received: January 27, 2020 | Published: April 22, 2020

\section{Introduction}

CDSHA usually considered being combat weapons, although doubts have been expressed about this (seeing for example. ${ }^{1,2}$ This is an important question, since their findings during the 19th and 20th centuries only on the territory of Western Eurasia are approaching 10 thousand..$^{3-5}$ Of particular interest in this regard is the territory of the Volga-Kama region, where by the end of the first half of the 20th century, several hundred of them were already been found. Next, we'll only talk about stone HA. They are dated IV-I thousand BC, and found in Europe (before the Pyrenees), Asia Minor, Northern and Middle Mesopotamia, the North Caucasus, in burials, hoards, or outside of them. The Eastern border of the finds is probably Western Siberia. The discrepancy between the declared and actual functions of the product can be detected by analyzing the morphology, material properties, color and decor of the CDSHA. The scope of work allows you to consider and analyze only the main factors listed.

\section{DHSA prehistory}

The main requirement for a combat DSHA is the strength that allows it to be used repeatedly without destruction. The most common form of this type of product is the blade and the butt connected by an "adapter-bushing". To motivate its appearance by the need to increase the impact strength at the location of the most vulnerable place, it is impossible to drill. The most effective and economical way to increase the impact strength of DSHA is to increase the thickness of the product in a dangerous section, which we see on wedge-shaped (working) TM (types 1-3, type 1-2, etc. The second common type of structural excesses on some types of DSHA is triangular protrusions made on the side faces of products, and" stretched " parallel to the drill (North Caucasian culture), according to Ingul culture.

They are found on axes of type 11 from the most ancient layers (№ 11-13) of the Ezero settlement, on a clay model from Tell Ukayr, types $\mathrm{D}, \mathrm{E}$ by Glob. These triangular protrusions sometimes turn into ledges, and the axe-hammer (in the plan) is transformed into an "iron", with a "butt-hammer "attached to it, turning into the "obushkovy" type (subtype B) according to visual identification of this transition zone ("center"), in which the drill itself is located, is sometimes achieved by applying color markers, for example, a circular rim of black color on a clay model from Tell Ukayr. The third type of dividing element is a protrusion on the upper edge of the hammer-axe ("chord" as defined by V. A. Gorodtsov, which is "stretched" from the tip to the butt. Sometimes this projection replaces the longitudinal narrow groove on the upper surface ("back") of the hammer-axe. However, these design solutions (adapter sleeve, "stiffeners", chord) lead to useless labor costs, and in no way increase the impact strength of the product.

Effective use of a stone axe when cutting wood and processing wood is possible only, when using a special cutting technique - applying frequent, but not strong blows, let's call it "woodpecker style". It is not accidental that the terms "axe"- pelecos and "woodpecker"pelecas are almost identical in ancient Greek. Strong blows with the point of a stone axe, led first to chips on the blade, the destruction of its edge, and later to the destruction of the product itself, primarily at the location of the drill. The impact strength of the product could be partially increased by selecting suitable materials with a special, feltlike microstructure (jade, diorite), which consequently gives them an increased impact strength. The term *tek's - axe, to make something with an axe (pre Indo-european), has satisfactory phonetic and morphological convergence with tasha - axe, tashan - creator (middle Avestan), dehsa - axe (old upper German), tashel - hew (Arminian), taksan - carpenter (old Indian.). "Tesh" - the name of the axe among mountain Tajiks and the Russian" tesat" - that is, working with a stone axe meant first of all not cutting, but hewing. It is difficult to imagine the use of such the military weapons. ${ }^{6}$

Clay models of drilled SHA also known. In Ezero (Bulgaria, Early and Middle Bronze), a relatively small (about 21\%) group that performed the functions of "combat" was also singled out among the many DSHA finds. The basis for this statement was their " pretentious form" and the fact of finding clay models of hammer axes of these types. The maximum of clay models was found in the tenth construction horizon, covering the time from the last quarter of IV thousand BC to the end of the early bronze age. Among the clay models, the form of long-bladed axes with dividing protrusions located on the side faces of the HA, next to the drill and the pestle-like (phalloid) end of the hammer part (type 11). According to Merpert N. Ya., forms of axeshammers connected with performance of "fighting, parade or even ritual functions" for modeling was chosen. The order of enumeration of the functions of axes leaves no doubt as to how priorities were set, it is clear that the ritual functions of stone axes-hammers are the author of the work Merpert N. Ya. Thought they were third-rate. In Ezero, clay models of hammer axes were made for a long time, up to the middle 
of MB age (the turn of the third and second thousand BC). And SHA "combat (ritual?)" assignments and their clay models at the second stage of the operation of the Ezero settlement (construction horizons № 6-1) practically disappear at the same time. On the monuments of the Fatyanovo culture, clay models found in children's graves. It is unnecessary to talk about their "combat" purpose. Some types of SHA (e.g. types 10, 11 according to Krainov D.A. have another, very characteristic feature of the form. It is most clearly manifested in the SHA from Kuban and the North Caucasus and others. We are talking about a bend," fracture "of the DSHA profile, when the blade and butt form an obtuse angle, resembling a "Chevron" (flattened arch), its "top" (bulge) is usually located on the axis of the drilled hole. We also note the discovery of a clay model of a hammer-axe in the lower reaches of the Irtysh (settlement Luchkino 1). The product had a phalloid end of the butt and a "fracture" of the profile, in this case, in solidarity with the phalloid completion on the clay models of the Fatyanovsky (Balanovsky burial ground) DSHA. This can serve as an indication that during its manufacture (Western Siberia, the late bronze age, the beginning of the first Millennium BC), either the skills of stone processing or access to the sources of the corresponding stone species were lost. But, despite this, in the mythologized consciousness of the inhabitants of the settlement, the most important part of the mythological ritual, realized in a special form of the product, has been preserved - implying a phalloid butt and a" fracture " of the HA profile. And finally, there are known finds of DSHA, with a blunted blade, they are especially frequent on monuments of catacomb culture (Figure 1). ${ }^{7}$

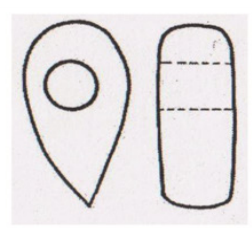

1.

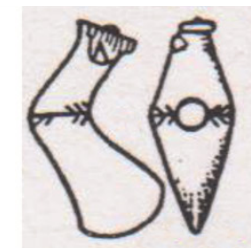

2.

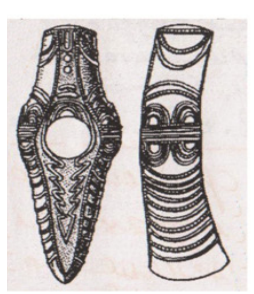

3.

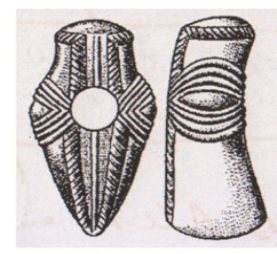

4.

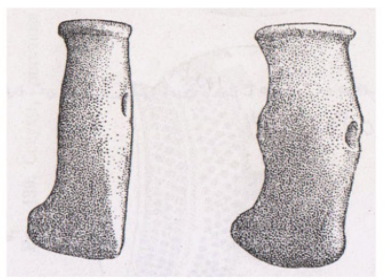

6.

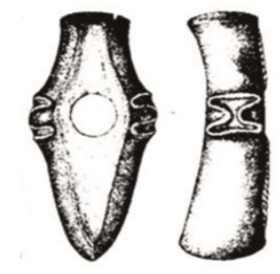

5.
As a result, it turns out that DSHA and their clay models from the monuments of the early and middle bronze age of Western Eurasia have a set of features that don't in any way improve the declared ("combat") functions. It is non-functional morphology, selection of the boundary between the blade and hammer butt, using the adapter ("sleeve"), the protrusions of triangular cross-section on the side faces, and the chord (in the form of ribs or grooves) on the upper horizontal face ("back") products, "stretched" from the blade to chlorite, and continuing, in some cases, next to the butt. Further, this is an inflection (fracture) of the TM profile at the location of the drill. Another, fastening DHSA only "the nozzle" on the handle, not provides stable exploitation, so stone axes as battlegroups, so and workers always drugged leather belts.

\section{DSHA technology, structure features, visua appearance and linguistics terms}

In the manufacture of the bulk of the DSHA, preference given to rocks of stone that combine different shades of green with a mottled surface texture (diorite, jadeite, serpentine). In this case, the coil has a low mechanical strength, which not combined, with its purpose as a shock weapon. Finally, DHSA often has the entire surface polished, not just the blade. To understand why this operation (in General functionally unnecessary if you put them the main function of use as tools or weapons), you need to remember the consequences of lightning in the stone rock or soil that is nearly always the formation of fulgurite,-“thunder thumb", which is a hollow glassy tube surrounded by compacted soil. The appearance of green color is due to the almost ubiquitous presence of iron oxides in the soil and stones. Iron replaces mainly magnesium, calcium, or aluminum ions in them, turning them green after melting. This makes clear the choice of rocks that have different shades of green, and the subsequent polishing of products in order to give them the Shine inherent in the vitreous state. These fulgurites (thunderstones) were the object of worship of many peoples of Eastern Europe, and Siberia. ${ }^{8}$

Selkups called them "fallen arrow from the sky" - nopkgyny alchypyl tischschya or "pyu " - devil's finger. "Agdi Suhinin" (Negid.), "agdi sevari" (Nanaj), - the axe of thunder (verbatim), the name of stone "arrows" that are found in trees split by lightning. Amur Nivkhs called stone axes "liy tukh" - thunder axes. Thunder stones ("sata") formed after lightning hit the ground, the Yakuts gave properties of influence on the weather. At the same time, L. J. Sternberg noted that the Ainu did not attribute them to a celestial origin, although stone tools were widely used in everyday life. The above allows us to assume that the idea of SKTM and the need to drill the stone (an operation that is completely unnecessary for practical tasks) for putting it on the handle was based on the "thunder finger" - fulgurite. In addition, the curved profile of a polished stone drilled hammer axe is a kind of counting "pair" (chevron), which also has a primordial connection with lightning and thunderstorms. Naturally, the different shape of the sides of this object, "blade-tip" and "hammer-butt" having different purposes, should have different semantics.

All of the above features of morphology, color, and decor are inherent in the four DHSA from the L collection (Troy IIg, 24002200 BC, State fine arts Museum) found by Schliemann G. Their detailed description (and bibliography of works dedicated to them) is in (see also the site http://www.antic-art.ru/data/troy/7_four_axes/ index.php), so for now we will limit ourselves to what is relevant to this work. The texture of the stone used-jadeite, lapis lazuli and

Figure I Combat drilled stone axe-hammers. 
jade-naturally reproduces the diversity of snake skin (blue-green background with patches of black, yellow and other colors). The surface of the products is polished to a mirror shine. Both parts-the blade and the butt are visually separated. In a jadeite axe, this is a "joint-barrel", in a lapis lazuli and jade, these are circular protrusions located on both sides of the drill, a kind of "adapter". All products have a noticeable profile fracture at the location of the drill. Protrusions are "stretched" from the blade to the drill, visually dividing the blade part into two symmetrical halves. Head of the axe parts have the shape of a slightly elongated oval. The length of the axes is $25.9-31 \mathrm{~cm}$ ("span"), the blades have an elaborate shape with extensions at the ends. The side faces of the products decorated with protruding "cones". There are remnants of gilding on the cones. The materials used can be considered traditional-these are rocks of different shades of green with a grainy and non-uniform texture. The material, morphology, and Polish completely corresponding to those described, are also found in four DHSA from the Borodino treasure. Their obvious similarity to isochronous products from Kuban and the North Caucasus, allowed us to express an opinion about them as some dynastic treasures passed down by inheritance. ${ }^{9,10}$

As for the decor, the DHSA decorated, first of all, with an adapter (or "bushing"). On Trojans, they are convex hemispheres. The billing information displayed by them has a quite distinct calendar character. For example, a jade TM from the 1 collection has $45(15 \times 3)$ cones on each side. This number is half of the average interval between solstices and equinoxes, such a breakdown of the annual calendar cycle consisting of 360 days is known in ancient Iranian and Greek and other calendar traditions. The lazurite TM from the 1 collection has forty-one cones $(2 \times 14+13)$ on each side face, which in total gives the number 82 , equal to three $(27,3 \times 3)$ sidereal lunar months, it is widely represented on artifacts from various archaeological cultures of Western Eurasia. On the drilled KTM of the Ingul culture (catacomb KIO), both the "adapter" and the "chord" (rarely the blade) can be decorated. It can be in the form of a meander, which combined with the sign "double axe" (eight) or "herringbone", consisting of chevrons.

\section{DHSA semantic}

The semantics of meanders and chevrons as elements that simulate bending can be correlated with the oldest counting "devices" (elbow, knee). This is probably why the close vocalization of the lexemes $\alpha \rho \theta \mu$ ov - joint (other Greek.) and $\alpha \rho i \theta \mu$ os-counting, is not accidental, and is due to their genetic relationship, and primarily due to the initial correlation of counting procedures with joints. If this is true, then some types of SKTM, especially those with a" bend "profile, can be correlated with "counting" devices, in favor of this may indicate a visual selection of the transition zone between the butt (hammer) and the blade (axe), or structurally (sleeve), or painting and/or decoration. The presence of two or more types of counting symbols on the product may be evidence of the existence of a certain calendar algorithm, or a degree of complexity inherent in this product. Decor on other types of DHSA, for example, fatyanovskih, though simpler (ribs), but still located on the side faces of the transition zone between the "axe" and "hammer".

\section{Conclusion}

Before DHSA correlate with a particular period of time, you need to remember that in different versions of the myth of the Indo-
European calendar, the winter season is correlated with the male (from the "top male" of moisture) fertilizing the ground, and the hot summer season with female - fruiting "conceived" of the land. Vedic poets identified the winter solstice with the Sky-Father (Dyaus) and the summer solstice with Prithivi (Mother Earth). Macrobius correlated winter with the male principle, believing the winter solstice to be the moment of Attis " death and the simultaneous birth of his son Papas. The terms attas and atti denote the concept of "father" in other Irish, ancient Indo-European languages of Asia Minor (Hittite, luvian, Lycian, etc.), as well as in Tocharian. Given the context, it is acceptable to correlate the obuch with the "heavenly" hammer, the mythologized cause of thunderstorms and thunder, and as a result of precipitation. In the realities of possible variants of the IndoEuropean homeland (Asia Minor-Northern Mesopotamia or SouthEastern Europe), this assumed a warm, rainy winter in the Atlantic [40, 81-83]. Then "blade-blade" can be correlated with the symbol of summer-heat, fire and feminine. If this is the case, then the edge of the blade becomes the border that divides the summer warm season ("big summer") into two parts, and this separator must be an event associated with thunder, lightning and heavy rains. These conditions are quite consistent with the day of Elijah (end of July or beginning of August), the time of the "turning point" of the weather to the winter. The drilled stone hammer-axe thus becomes a symbol of the threeseason (winter and two halves of summer) model year.

The transition zone on ritual stone axes-hammers is almost always allocated in the form of a projection (roller), a ring belt, a "barrel", a circular notch connecting two parts of the binary system-heat-cold, moisture-dry, female-male, being, thus, a joint, a" joint" of a special kind with a peculiar semantics. For example, the other Indus. "Parvan" had the meaning of " joint, bend, turning point of time", thus having the semantics of the calendar counting unit.

\section{Acknowledgments}

None.

\section{Funding}

None.

\section{Conflicts of interest}

Author declares that there is no conflict of interest.

\section{References}

1. Degen BE. Barrows in the Kabardian Park of Nalchik. MIA. 1941;3:213-316.

2. Tsyb SV. Stone battle axes of Western Siberia. Military Affairs of ancient tribes of Siberia and Central Asia. Novosibirsk. 1981; 6-13.

3. Bryusov AYa, Zimina MP. Stone drilled battle axes on the territory of the European part of the USSR. Archeology of the USSR, SAI. 1966;B4-4:98.

4. Malmer M.P. The Neolithic of South Sweden. TRB, GRK and STR MP Malmer. Stockholm. 2002. 284 p.

5. Efimov AM. Drill hammers from the collection of the State Museum of the Tatar ASSR. Moscow. 1951;26:170-176.

6. Merpert NI. Ritual of the model axes from Ezero. Monuments of ancient history of Eurasia. Moscow; 1975. p. 163-172. 
7. Kushnareva K. H. The Bronze Age of the Caucasus and Central Asia. Part 1. Early and middle bronze age in the Caucasus/Kushnareva K. Kh. 1994.384 p.

8. Sharafutdinova IM. Ornamentovany of sakiri-hammers in catacombnikh pohovan on Ingul. Archaeology. 1980;33:60-70.
9. Lloyd S. Tell Ugair. Report on the Excavation. JNES. 1943;2(2):135-158.

10. Gorodtsov VA. Culture of the bronze age in Central Russia. Moscow; 1916. $228 \mathrm{p}$ 